\title{
Pediatria
}

http://www.revistapediatria.org/

Regional Bogotá

DOI: https://doi.org/10.14295/rp.v53i3.145

Reportes de caso

\section{Hidrocolpos Neonatal}

\author{
Rubén Coca Pasapera ${ }^{a}$, Liz Arlet Elera Romero ${ }^{a}$, Jesús Ramírez Rivera ${ }^{a}$, Víctor Peralta Chávez ${ }^{b}$, \\ Luz Cisneros Infantas ${ }^{c}$ \\ a Estudiante de Medicina, Escuela de Medicina, Facultad de Medicina Humana, Universidad Privada Antenor Orrego, Trujillo, Perú. \\ b Pediatra, Departamento de Neonatología, División de UCI neonatal, Hospital Belén de Trujillo, Trujillo, Perú. \\ c Pediatra, Departamento de Neonatología, Hospital Belén de Trujillo, Trujillo, Perú.
}

\section{INFORMACIÓN DEL ARTÍCULO}

Historia del artículo:

Recibido el 05 de julio de 2019

Aceptado el 07 de noviembre de 2020

Palabras clave:

Hidrocolpos

ano imperforado

recién nacido

ultrasonido

anomalías congénitas.

\section{R E S U M E N}

\begin{abstract}
Antecedentes: El hidrocolpos neonatal es una patología infrecuente caracterizada por dilatación y acumulación de líquido en el canal vaginal como resultado de obstrucciones vaginales congénitas. La utilización de ecografía es un medio de diagnóstico importante para brindar un tratamiento oportuno y disminuir las complicaciones que conlleva. Reporte de caso: Recién nacida de 39 semanas presentó ano imperforado y abdomen distendido. Se realizó ecografía abdominal evidenciándose hidrocolpos e hidroureteronefrosis bilateral moderada. Dentro de los hallazgos de laboratorio se evidenciaron: falla renal, hiponatremia, y trastorno de coagulación que fue corregido oportunamente. Fue intervenida quirúrgicamente realizándose colpotomía y drenaje de hidrocolpos, evidenciando posteriormente en ecografía control desaparición de este. Se dio el alta a la paciente con 28 días de vida por presentar una evolución satisfactoria. Conclusión: Se debe tener alta sospecha de hidrocolpos ante una masa a nivel de pelvis en una recién nacida. El diagnóstico precoz mediante la ecografía es importante para la prevención de complicaciones obstructivas y renales que aumentan la morbimortalidad de las pacientes.
\end{abstract}

\section{Neonatal hydrocolpos}

A B S T R A C T

Background: Neonatal hydrocolpos is a rare pathology characterized by dilation and accumulation of fluid in the vaginal canal due to congenital vaginal obstructions. The use of ultrasound is an essential diagnostic means to provide timely treatment and reduce its complications. Case report: A 39-week-old newborn presented an imperforate anus and a distended abdomen. An abdominal ultrasound was performed, showing hydrocolpos and moderate bilateral hydroureteronephrosis. Among the laboratory findings, renal failure, hyponatremia, and coagulation disorder were diagnosed, which was timely corrected. She underwent surgery, performing colpotomy and drainage of hydrocolpos, later showing its disappearance on ultrasound. The patient was discharged at 28 days of age due to a satisfactory evolution. Conclusion: Hydrocolpos should be highly suspected of a mass at the pelvis level in a newborn. Early diagnosis by ultrasound is essential for preventing obstructive and renal complications that increase the morbidity and mortality of patients.

\footnotetext{
${ }^{*}$ Autor para correspondencia. Jesús Ramírez Rivera Correo electrónico: jramirezr4@upao.edu.pe, jesusrr9441@gmail.com
}

Como Citar: Hidrocolpos Neonatal. Coca Pasapera R, Elera Romero LA, Ramírez Rivera J, Peralta Chávez V, Cisneros Infantas L. Pediatr. 2020;53(3):111114. 


\section{Introducción}

El hidrocolpos neonatal es la dilatación quística del canal vaginal caracterizado por la acumulación de líquido en su interior. Se trata de una alteración del tracto urogenital poco frecuente con una incidencia de 1 en 16000 a 1 en 30000 nacidas vivas $(1,2)$.

Su etiología es variada y tiene explicación por alteraciones en el desarrollo embrionario: interrupción de la canalización del intestino posterior, como el seno urogenital y la cloaca persistentes (ej.: himen imperforado y fistula uretrovaginal), una falla en la canalización vaginal, comúnmente a nivel distal, como septo o tabique vaginal, y una virilización de los genitales externos por hiperplasia suprarrenal congénita $(3,4)$.

Existen pocos casos reportados en la literatura, motivo por el cual se tiene el objetivo de presentar un caso de una recién nacida con hidrocolpos que fue diagnosticada y tratada de forma oportuna con una evolución favorable, para poder así, afianzar conocimientos sobre esta patología.

\section{Descripción del caso}

Se presenta el caso clínico de una recién nacida de 39 semanas producto de parto por cesárea, con peso al nacer de 2870 gr, talla de $45 \mathrm{~cm}$, Apgar de 5 y 7 puntos. Hija de madre de 20 años, sin antecedentes patológicos personales. Cinco consultas prenatales, en donde se evidenció polihidramnios, y dextrocardia.

Ingresó a la unidad de cuidado intensivo (UCI) neonatal por sepsis neonatal y síndrome dismórfico, con sonda orogástrica a gravedad, abdomen distendido +/+++, depresible y ano imperforado. Se le realizó ecografía abdominal evidenciándose hidroureteronefrosis moderada bilateral (Figura 1) más hidrocolpos (Figura 2).

Se realizaron exámenes auxiliares, teniendo como resultados: creatinina de $2.04 \mathrm{mg} / \mathrm{dl}$, Na de $131.6 \mathrm{mmol} / \mathrm{L}$, tiempo de protrombina (TP) de 32 segundos, tiempo de protrombina parcial (TPT): 76 segundos, international normalized ratio (INR) de 3.2, Proteína C reactiva (PCR) de $13.5 \mathrm{mg} / \mathrm{L}$. Evidenciándose alteración de la función renal, hiponatremia y trastorno de coagulación, que fue corregido con vitamina $\mathrm{K}$ y transfusión de plasma fresco congelado.

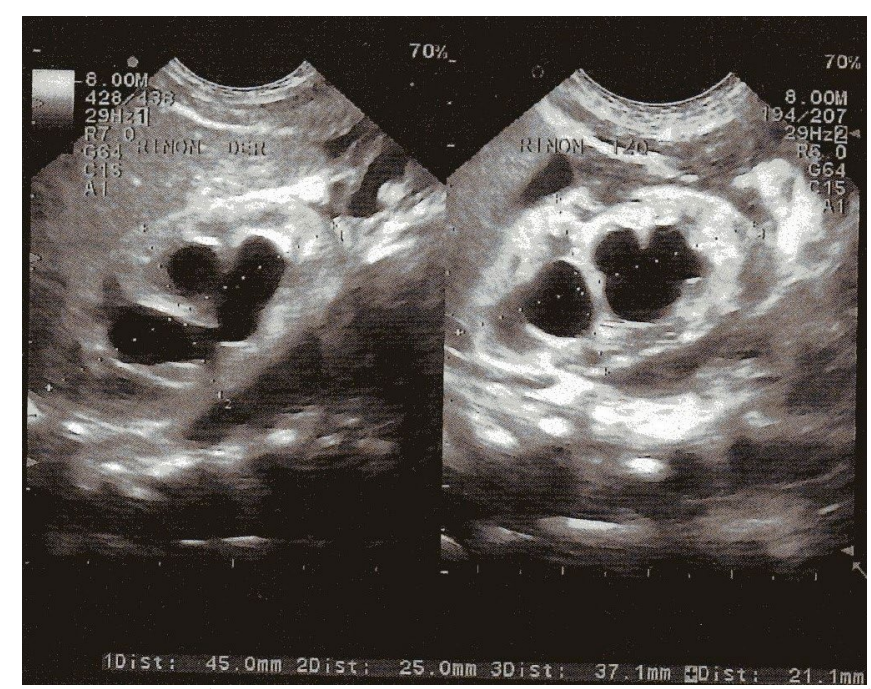

Figura 1. Riñón derecho: De 45 x 37 mm. Riñón izquierdo: De 37.1 x $21.1 \mathrm{~mm}$. Ambos con parénquima homogéneo, contornos regulares. Se visualiza moderada dilatación pielocalicial que se extiende a uréter, no cálculos.

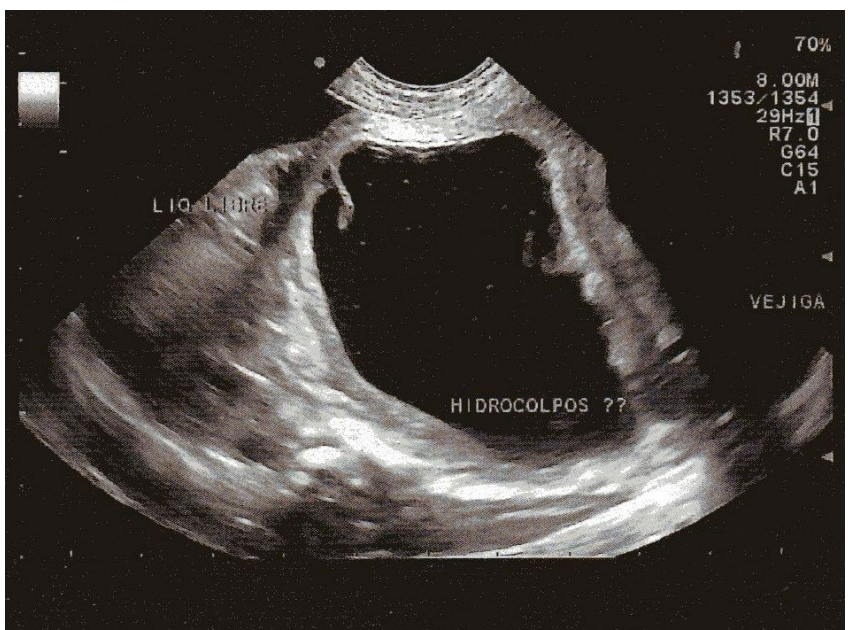

Figura 2. Se aprecia líquido de 70 x $30 \mathrm{~mm}$ (DL x DAP) retrovesical que impresiona estar dentro de cavidad uterina. 
Fue intervenida quirúrgicamente a las 24 horas de vida, mediante una laparotomía exploratoria evidenciándose estructura dilatada con cantidad de líquido claro y detritos blanquecinos que se comunica con el útero, vejiga de paredes engrosadas, colon descendente y sigmoides dilatado. Se le realizó una colostomía usando técnica de Mikulicz y drenaje del hidrocolpos.

Al quinto día de vida, continuó con distensión abdominal, además se evidenciaron ruidos hidroaéreos (RHA) disminuidos, se canalizó orificio único sin obtención de orina, por lo que se planteó cloaca persistente más permanencia de hidrocolpos y reingresó a sala de operaciones encontrando salida de líquido mucoide de aproximadamente $80 \mathrm{cc}$, por lo que se dejó con sonda Foley $\mathrm{N}^{\circ} 14$ permanente en saco vaginal, realizándose colpotomía y drenaje, teniendo cobertura antibiótica con Ampicilina y Amikacina.

A los 11 días de vida, se realizó una ecografía abdominal de control, demostrando desaparición de la dilatación vaginal (hidrocolpos) (Figura 3), e hidronefrosis bilateral leve.
A los 18 días de vida, continuó con nutrición enteral por sonda orogástrica, con bolsas de colostomía y colpotomía funcionales, afebril y hemodinámicamente estable por lo que se suspendió antibioticoterapia. Al día 22 es transferida a la Unidad de Cuidados Intermedios por tener una evolución favorable.

A los 28 días de vida, al encontrarse clínicamente estable y responder adecuadamente a las medidas terapéuticas brindadas, fue dada de alta, por lo que se programó su posterior control por consultorio externo de Pediatría.

\section{Discusión}

La aparición de hidrocolpos puede generarse debido a algún tipo de obstrucción vaginal congénita la cual puede ser principalmente de dos tipos: 1) Asociado a himen imperforado y 2) Malformación cloacal, siendo este último tipo el más frecuente y a su vez el que presenta la paciente de este caso. Es impor-

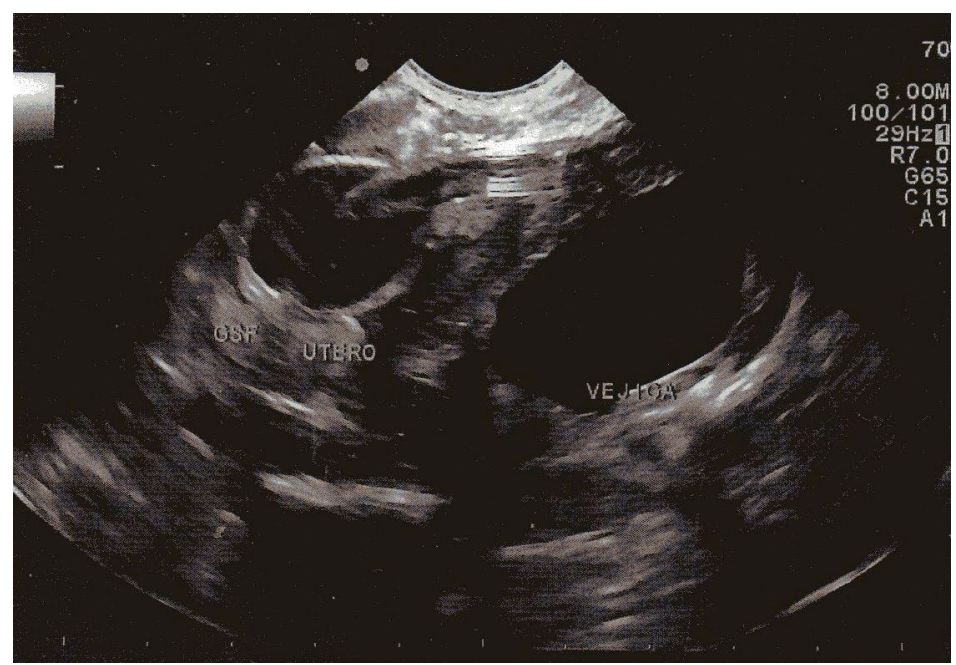

Figura 3. Vejiga de mediana repleción. No formaciones tumorales ni litiasis. Presencia de globo de sonda Foley en cavidad uterina sin presencia de contenido líquido en el interior de la cavidad.

tante resaltar el hecho de que aunque su incidencia sea muy rara y no se tengan datos precisos sobre la misma, siempre que veamos la presencia de una masa a nivel de pelvis en una recién nacida debemos orientar el plan diagnóstico hacia un hidrocolpos (5).

Por otra parte, en el caso también se señala la presencia de sepsis neonatal y esto es, debido a que el hidrocolpos al ser una dilatación quística de la vagina puede generar la acumulación de líquidos y secreciones vaginales, con la consecuente infección (6).

Dentro de los procedimientos diagnósticos, sin lugar a dudas el más importante es el empleo de la ecografía, pudiéndose evidenciar una masa quística con mala visualización de la vejiga, además de la presencia de detritus y de hidronefrosis debido a la obstrucción que el hidrocolpos produce, lo que también se observa en la paciente del caso, que incluso llega a tener hidronefrosis bilateral (7).
En cuanto al tratamiento, cabe señalar que el hidrocolpos se asocia a otras malformaciones dentro de las cuales se encuentra el ano imperforado y que también presenta la paciente de este caso, razón por la cual se debe evaluar el tratamiento quirúrgico para su manejo. Sin embargo, el primer paso para el tratamiento de un hidrocolpos es el drenaje, y este es muy importante si se hace precozmente ya que ayuda a prevenir o disminuir la hidronefrosis y obstrucción gastrointestinal. Lo que se observó en el presente caso, es que a la paciente se le realizó una colpotomía y drenaje, desapareciendo el hidrocolpos, pero persistiendo de forma leve la hidronefrosis bilateral (8).

\section{Conclusiones}

El hidrocolpos es una patología infrecuente, sin embargo, debemos tener un alto índice de sospecha ante la presencia de 
una masa a nivel de pelvis en una recién nacida. El diagnóstico temprano y oportuno mediante el uso de la ecografía es crucial para la prevención de complicaciones obstructivas y renales que puedan llevar a la muerte de la paciente, siendo de vital importancia el manejo multidisciplinario para un adecuado tratamiento, en este caso quirúrgico.

\section{R E F E R E N C I A S}

1. Khanna K, Sharma S, Gupta D. Hydrometrocolpos etiology and management: past beckons the present. Pediatric Surgery International. 2017; 34(3):249-61.

2. Rodriguez R, Delgado R, Alvarado M, Garcia J. et al. Fetal hydrometrocolpos and congenital imperforate hymen: Prenatal and postnatal imaging features. Journal of Clinical Ultrasound. 2018; $1-4$

3. Garfias C, Barrera A, Zubieta R, Ugarte F. Hidrocolpos e . hidrometrocolpos neoanatal: a propósito de un caso clínico. Rev Chil Pediatría. 2011;82(2):137-41.

4. Sarmiento Y, Crespo A, Sánchez I, Menéndez Y. Hidrocolpos neonatal. Rev Cuba Pediatría. 2009;81(4):93-7.

5. 5. Letelier N., Vigneaux L., Celis S. et al. Enfrentamiento clínico del hidrocolpos a diferentes edades pediátricas. Rev Chil Urol. 2013;78(4):40-2.

6. Unda S, Unda J, Arizmendi J, Alfaro K. Himen imperforado con hidrocolpos. An Med Mex.2016;61(1):44-47.

7. Madero G., Echeverry L., Jiménez Y. Diagnóstico neonatal de hidrometrocolpos. Pediatr. 2015;48(3):75-9.

8. Speck K., Arnold M., Ivancic V., Teitelbaum D. Cloaca and hydrocolpos: laparoscopic-, cystoscopic- and colposcopicassisted vaginostomy tube placement. J Pediatr Surg. 2014;49(12):1867-9. 